\title{
MiR-4505 aggravates lipopolysaccharide-induced vascular endothelial injury by targeting heat shock protein A12B
}

\author{
$\mathrm{XU} \mathrm{ZHANG}^{1 *}$, YI CHEN ${ }^{1 *}$, LEI WANG $^{1 *}$, QIUXIANG KANG ${ }^{1}$, GUIFANG YU $^{2}$, \\ XIAOJIAN WAN ${ }^{1}$, JIAFENG WANG ${ }^{1}$ and KEMING ZHU ${ }^{1}$ \\ ${ }^{1}$ Department of Anesthesiology and Intensive Care Medicine, Changhai Hospital, \\ The Second Military Medical University, Shanghai 200433; ${ }^{2}$ Department of Anesthesiology, \\ The Third People's Hospital, Shanghai Jiaotong University, Shanghai 201999, P.R. China
}

Received November 13, 2016; Accepted October 10, 2017

DOI: $10.3892 / \mathrm{mmr} .2017 .7936$

\begin{abstract}
Heat shock protein family A member 12B (HSPA12B) is a heat shock protein primarily expressed in endothelial cells. Our previous study showed that it was protective against endothelial injury induced by lipopolysaccharide (LPS). The present study was performed to investigate whether micro (mi)RNA was involved in HSPA12B expression in endothelial cells challenged by LPS. We first screened the miRNA candidates potentially related to HSPA12B by bioinformatics analysis. Then the mimics of the miRNA candidates were transfected into human umbilical vein endothelial cells (HUVECs) to investigate the miRNAs that negatively regulated HSPA12B expression. The miRNA expression was also determined in LPS-stimulated HUVECs. Dual luciferase activity assay was performed to confirm the relationship between the candidate miRNA and HSPA12B. Role of nuclear factor (NF)- $\kappa \mathrm{B}$ in the miRNA expression was investigated by using its inhibitor. Finally, the role of the miRNA on LPS induced injury was investigated. Eleven miRNAs were screened by bioinformatics analysis and 4 of them could inhibit HSPA12B expression at both mRNA and protein levels. Among the 4 miRNA candidates, only miR-4505 was highly expressed in HUVECs stimulated by LPS. Luciferase analysis showed that miR-4505 directly interacted with the 3'untranslated region of HSPA12B. LPS-induced upregulation of miR-4505 was blocked by NF- $\mathrm{B}$ inhibitor. Transfection with miR-4505 mimics reduced the transendothelial electrical resistance and vascular endothelial-cadherin expression. The
\end{abstract}

Correspondence to: Professor Keming Zhu or Dr Jiafeng Wang, Department of Anesthesiology and Intensive Care Medicine, Changhai Hospital, The Second Military Medical University, 168 Changhai Road, Shanghai 200433, P.R. China

E-mail:kmzhu@aliyun.com

E-mail: jfwang@smmu.edu.cn

${ }^{*}$ Contributed equally

Key words: HSPA12B, heat shock protein family A member 12B, miR-4505, endothelial injury, lipopolysaccharide scratch test demonstrated that miR-4505 inhibited endothelial migration capacity. In conclusion, miR-4505 downregulates the expression of HSPA12B and aggravates the LPS-induced vascular endothelial cell injury.

\section{Introduction}

Sepsis and its complications are still serious clinical problems (1). Lung injury (ALI) remains the common complication of sepsis (2). Unfortunately, development of effective pharmacologic and ventilatory treatment strategies for sepsis-induced lung injury has not made significant progress over the past several decades. Pulmonary microvascular endothelial cells play an important role in pulmonary blood barrier. They are involved in the transportation of nutrients and metabolites in the lung tissue, inflammatory response and endothelial regulation (3). Many studies have shown that PMVECs were destroyed during sepsis-induced lung injury, thus leading to increased permeability of pulmonary blood barrier and then activation of the coagulation system and inflammatory response system (4). Therefore, PMVECs is essential to reduce lung injury in sepsis.

HSPA12B, a newly discovered member of HSP70 superfamily, is predominately expressed in vascular endothelial cells and participates in vascular generation and migration (5). Zhou et al reported that upregulation of HSPA12B could attenuate sepsis-induced cardiac dysfunction (6). HSPA12B was also demonstrated to improve cardiac function and remodeling after myocardial infarction via eNOS-dependent mechanism (7). In vitro, Wu et al (8) found that HSPA12B could inhibit LPS-induced inflammatory response in HUVECs. Our previous study showed that HSPA12B was upregulated in LPS-stimulated endothelial cells and it was defensive against LPS induced impairment of endothelial permeability (9). Therefore, HSPA12B may be an intrinsic protein mediating endothelial protection against inflammatory insult, but it remains unclear how HSPA12B was upregulated. Post-transcriptional regulation may be involved in the regulation of HSPA12B gene expression; however, the underlying mechanism has not been well understood.

miRNA-mRNA interaction is one of the most common mechanisms of post-transcriptional regulation $(10,11)$. One miRNA can act on a variety of mRNAs and an mRNA may 
also be affected by multiple miRNAs $(12,13)$. Currently there are limited data that investigate the role of miRNA in the regulation of HSPA12B and the function of vascular endothelial cells. In the present study, we screened several miRNAs potentially correlated with HSPA12B by bioinformatics analysis and performed in vitro experiments to identify the candidate miRNAs targeting at the 3'UTR of HSPA12B mRNA.

\section{Materials and methods}

Reagents. Escherichia coli LPS was purchased from Sigma-Aldrich (St. Louis, MO, USA), and the NF- $\kappa \mathrm{B}$ inhibitor were obtained from Calbiochem (catalog number 481406, EMD Chemicals, San Diego, CA, USA). Liposome Lipofectamine 2000 was purchased from Invitrogen (Carlsbad, CA, USA). Human anti-HSPA12B antibody was from Abcam (Cambridge, UK); anti- $\beta$-actin antibody, goat anti-rabbit IgG-HRP secondary antibodies, and rabbit anti-mouse IgG-HRP secondary antibodies were from Cell Signaling Technology (Danvers, MA, USA). VE-cadherin antibody was obtained from R\&D Systems Inc (Minneapolis, MN, USA).

Bioinformatics analysis. The miRNAs potentially related to human HSPA12B were searched in the TargetScan (http://www.targetscan.org/), miRbase (http://www.mirbase.org/) and PicTar (http://pictar.mdc-berlin.de/) databases.

Cell culture and transfection. HUVEC was cultured in DMEM high glucose medium (Hyclone, Logan, UT, USA), supplemented with $10 \%$ fetal bovine serum at $37^{\circ} \mathrm{C}$ with $5 \%$ $\mathrm{CO}_{2}$. Transfection was performed using Lipofectamine 2000 (Invitrogen, CA, USA) according to the manufacturer's instruction. After the cells reached $80 \%$ confluency, $10 \mathrm{nM}$ miRNA mimic or negative control (Genepharma, Shanghai, China) were transfected into HUVECs by using Lipofectamine 2000. The transfection mixture was incubated for $6 \mathrm{~h}$. Negative control mimics were transfected as matched controls.

Real-time PCR. Total RNA containing miRNA was extracted from HUVECs using Trizol reagent (Invitrogen). Primers used for the polymerase chain reaction (PCR) were designed to be isoform specific (Table I). Following instructions of the Rever Tra Ace qPCR RT Kit (Toyobo, Osaka, Japan), cDNA was obtained by reverse transcription $\left(30 \mathrm{~min}\right.$ at $37^{\circ} \mathrm{C}$ and $5 \mathrm{~min}$ at $98^{\circ} \mathrm{C}$ ) from $1 \mu \mathrm{g}$ purified total RNA. To quantify the expression level of mRNA of HSPA12B, semi-quantitative PCR system was set up using $2 \mu \mathrm{l}$ of 10x PCR Buffer, $1 \mu \mathrm{l}$ of dNTP at $2.5 \mathrm{mM}$, $1 \mu \mathrm{l}$ of $\mathrm{Mgcl}_{2}$ at $25 \mathrm{mM}, 1 \mu \mathrm{l}$ of forward and reverse primers at $10 \mu \mathrm{mol} / 1,1 \mu \mathrm{l} \mathrm{cDNA}$, Taq enzyme and $0.25 \mu \mathrm{l}$ deionized water to constitute a total volume of $20 \mu \mathrm{l}$. The thermal cycle for amplification were as follows: (1) $94^{\circ} \mathrm{C}$ for $3 \mathrm{~min}$; (2) 28 cycles of denaturation, each consists of $30 \mathrm{sec}$ at $94^{\circ} \mathrm{C}, 45 \mathrm{sec}$ at $58^{\circ} \mathrm{C}$ and $20 \mathrm{sec}$ at $72^{\circ} \mathrm{C}$ followed by (3) a final extension for $7 \mathrm{~min}$ at $70^{\circ} \mathrm{C}$. GAPDH were used as internal control. PCR products were semi-quantified by electrophoresis in $1.5 \%$ agarose gel.

Western blot. The cells were washed twice with ice cold PBS and lysed with cell lysis buffer RIPA. Protein concentrations of the cell lysate extracts were measured with BCA assay (Pierce, Rockford, IL, USA) and equalized with the extraction reagent.
Equal amount of the extracts were loaded and subjected to SDS-PAGE, transferred onto PVDF membranes, and blocked with $5 \%$ non-fat milk in TBST, blotted with the antibody against HSPA12B (1:1,000), and subsequently secondary antibody $(1: 2,000)$. The bands on the membrane were visualized with an enhanced chemiluminescence (ECL) kit (GE, Amersham, UK). $\beta$-actin was used as an internal loading control.

Luciferase activity assay. 293 cells were seeded in 24-well plates. After the cells reached $60 \%$ confluency, the mixture with $0.5 \mu \mathrm{g}$ pGL3-HSPA12B-3'UTR plasmid (provided by Obio, Shanghai, China), $0.5 \mu \mathrm{g}$ mutant pGL3-HSPA12B-3'UTR plasmid (provided by Obio, Shanghai, China) and $50 \mathrm{pmol}$ miRNA mimic or negative control (Genepharma, Shanghai, China) were incubated with Lipofectamine 2000 (Invitrogen, CA, USA). After $24 \mathrm{~h}$, cells were collected and lysed with $10 \mu 1$ Luciferase Assay Substrate (Invitrogen, CA, USA), and then $10 \mu \mathrm{l}$ cell lysate supernatant was added. Luciferase assays were measured using Luminometer (E1910, Promega). And the luciferase activity was normalized by Renilla luciferase intensity.

Flow cytometry. Cells were removed from culture dish with trypsin and harvested by centrifugation. Antibody for flow cytometry was added and reaction was conducted in the dark for $30 \mathrm{~min}$. After centrifuging, the supernatant was discarded and 1xPBS was added. Total VE-cadherin expression was detected using a flow cytometer (BD FACSCalibur, Franklin Lakes, NJ, USA).

Transendothelial electrical resistance (TEER). Transendothelial electrical resistance (TEER) of HUVEC monolayer was determined using MERSSTx01 Electrode according to the instruction manual of the manufacturer (EMD Millipore Corporation, Billerica MA, USA). $0.4 \mu \mathrm{m}$ fibronectin-coated Transwell filters was used and $1 \times 10^{5}$ HUVECs were seeded on each filter until full confluence. After cell culturing of $72 \mathrm{~h}$, MERSSTx01 electrode was used to detect the TEER value of HUVECs sequentially and the mean was expressed in the common unit $\left(\mathrm{V} \mathrm{cm}^{2}\right)$ after subtraction of the value of a blank cell-free filter.

Scratch test. HUVECs $\left(5 \times 10^{5}\right.$ cells per well) were cultured $24 \mathrm{~h}$ in a 6 -well plates. A wound was made by craping monolayer cells with a $200 \mu \mathrm{l}$ pipette tip. Then the wounded cell monolayers were washed with serum-free medium. The medium was replaced with serum-free DMEM. The migration path of cell was tracked at 0 and 24 h using Olympus microscope and representative scratch zones were photographed.

Statistical analysis. The results were presented as the mean \pm SD. Comparison of mean between groups was performed using one-way analysis of variance (ANOVA). $\mathrm{P}<0.05$ was considered to be significant. GraphPad Prism5 (La Jolla, USA) was used for all statistical analysis.

\section{Results}

miRNA expression features in HUVECs stimulated with $L P S$. A total of 11 miRNAs were identified to potentially 
Table I. Primer sequences for RT-qPCR.

\begin{tabular}{|c|c|c|}
\hline Gene & Forward 5'-3' & Stem-Loop primer 5'-3' \\
\hline miRNA universal reverse primer & TGGTGTCGTGGAGTCG & \\
\hline hsa-miR-1224-3p & $\begin{array}{l}\text { ACACTCCAGCTGGGCCCCACC } \\
\text { TCCTCT }\end{array}$ & $\begin{array}{l}\text { CTCAACTGGTGTCGTGGAGTCGGCAATTC } \\
\text { AGTTGAGCTGAGGAG }\end{array}$ \\
\hline hsa-miR-3127-5p & $\begin{array}{l}\text { ACACTCCAGCTGGGATCAGGG } \\
\text { CTTGTGGA }\end{array}$ & $\begin{array}{l}\text { CTCAACTGGTGTCGTGGAGTCGGCAATTC } \\
\text { AGTTGAGCTTCCCAT }\end{array}$ \\
\hline hsa-miR-3657 & $\begin{array}{l}\text { ACACTCCAGCTGGGTTAGTGG } \\
\text { TTATTA }\end{array}$ & $\begin{array}{l}\text { CTCAACTGGTGTCGTGGAGTCGGCAATTC } \\
\text { AGTTGAGACACAGGG }\end{array}$ \\
\hline hsa-miR-4505 & $\begin{array}{l}\text { ACACTCCAGCTGGGAGGCAG } \\
\text { GGTC }\end{array}$ & $\begin{array}{l}\text { CTCAACTGGTGTCGTGGAGTCGGCAATTC } \\
\text { AGTTGAGTCCGACCC }\end{array}$ \\
\hline hsa-miR-3665 & $\begin{array}{l}\text { ACACTCCAGCTGGGGCGGCG } \\
\text { GGGC }\end{array}$ & $\begin{array}{l}\text { CTCAACTGGTGTCGTGGAGTCGGCAATTC } \\
\text { AGTTGAGTCGTCCAC }\end{array}$ \\
\hline hsa-miR-4736 & $\begin{array}{l}\text { ACACTCCAGCTGGGGTCGGG } \\
\text { TCTAT }\end{array}$ & $\begin{array}{l}\text { CTCAACTGGTGTCGTGGAGTCGGCAATTC } \\
\text { AGTTGAGTCCGTCCA }\end{array}$ \\
\hline hsa-miR-940 & $\begin{array}{l}\text { ACACTCCAGCTGGGCCCCTC } \\
\text { GCCCCCG }\end{array}$ & $\begin{array}{l}\text { CTCAACTGGTGTCGTGGAGTCGGCAATTC } \\
\text { AGTTGAGTTCCGTCC }\end{array}$ \\
\hline hsa-miR-4692 & $\begin{array}{l}\text { ACACTCCAGCTGGGTAGACT } \\
\text { ATGGGTGT }\end{array}$ & $\begin{array}{l}\text { CTCAACTGGTGTCGTGGAGTCGGCAATTC } \\
\text { AGTTGAGAGTCCGTC }\end{array}$ \\
\hline hsa-miR-4514 & $\begin{array}{l}\text { ACACTCCAGCTGGGAAGGG } \\
\text { GTTAG }\end{array}$ & $\begin{array}{l}\text { CTCAACTGGTGTCGTGGAGTCGGCAATTC } \\
\text { AGTTGAGTGTCCGTC }\end{array}$ \\
\hline hsa-miR-4742-5p & $\begin{array}{l}\text { ACACTCCAGCTGGGAGACA } \\
\text { TTTATAGGGA }\end{array}$ & $\begin{array}{l}\text { CTCAACTGGTGTCGTGGAGTCGGCAATTC } \\
\text { AGTTGAGAGTCCGTT }\end{array}$ \\
\hline hsa-miR-3664-3p & $\begin{array}{l}\text { ACACTCCAGCTGGGTTGAG } \\
\text { ACAGAAATG }\end{array}$ & $\begin{array}{l}\text { CTCAACTGGTGTCGTGGAGTCGGCAATTC } \\
\text { AGTTGAGAGAGTCCT }\end{array}$ \\
\hline U6 Forward primer & CTCGCTTCGGCAGCACA & \\
\hline U6 Reverse primer & AACGCTTCACGAATTTGCGT & \\
\hline
\end{tabular}

Hsa, homo sapiens; miR, microRNA; RT-qPCR, reverse transcription quantitative polymerase chain reaction.

target the 3'UTR region of HSPA12B mRNA, including miR-3657, miR-642a, miR-4505, miR-3665, miR-4736, miR-940, miR-4692, miR-4514, miR-4742-5p, miR-4269 and miR-3664-3p. In order to further establish the relationship between HSPA12B and the miRNAs screened by the bioinformatics analysis, the miRNA expression levels were determined by RT-PCR in the LPS-stimulated HUVECs. As a result, miR-3657, miR-4692, miR-4742 and miR-4505 were all upregulated in HUVECs at $12 \mathrm{~h}$ after LPS stimulation $(\mathrm{P}<0.05)$ (Fig. 1). The expression level of miR-4505 was highest, with a changing level more than 3 compared with $0 \mathrm{~h}$.

miRNA-4505 inhibits HSPA12B expression in LPS-challenged HUVECs. The HUVECs were transfected with the miRNA mimics of the 4 miRNA candidates as well as the negative control (NC). The RT-PCR and western blot assays showed that the expression levels of HSPA12B in the HUVECs transfected with the mimics of miR-3657, miR-4692, miR-4742-5p and miR-4505 were lower than those transfected with the negative control (Fig. 2A-C). In addition, in our previous research, HSPA12B was elevated in $12 \mathrm{~h}$ upon the LPS stimulation (5). We suspected that miR-4505 may negatively regulate the expression of HSPA12B. Therefore, miR-4505 was screened for further investigation as a potential candidate regulating the expression of HSPA12B in LPS-induced HUVECs.

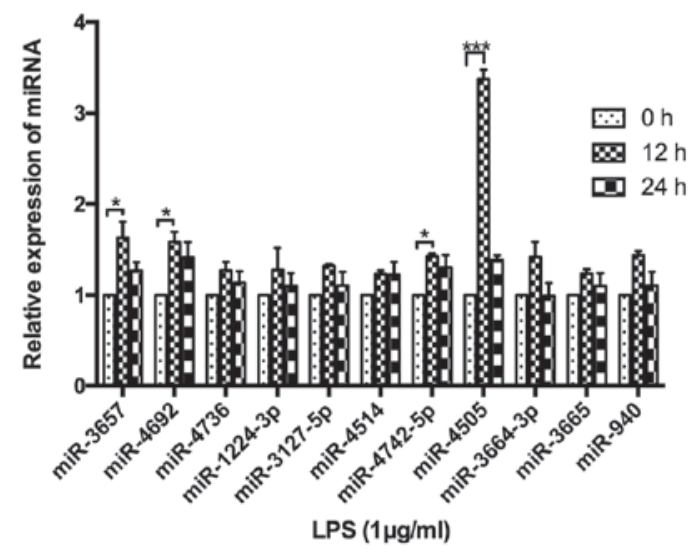

Figure 1. Changes of miRNA expression in HUVECs after LPS stimulation. HUVECs were transfected with miRNA mimics, then exposed to LPS for 12 and $24 \mathrm{~h}$. Expression of miRNA was detected by RT-qPCR. The results are mean $\pm \mathrm{SD}$ from three independent experiments. ${ }^{*} \mathrm{P}<0.05,{ }^{* * *} \mathrm{P}<0.001$ HUVECs, human umbilical vein endothelial cells; miRNA, microRNA; RT-qPCR, reverse transcription quantitative polymerase chain reaction; LPS, lipopolysaccharide.

miR-4505 directly targets at 3'UTR of HSPA12B mRNA. Luciferase analysis was performed to investigate the relationship between HSPA12B and miR-4505. To obtain direct evidence that miR-4505 targets HSPA12B mRNA, 

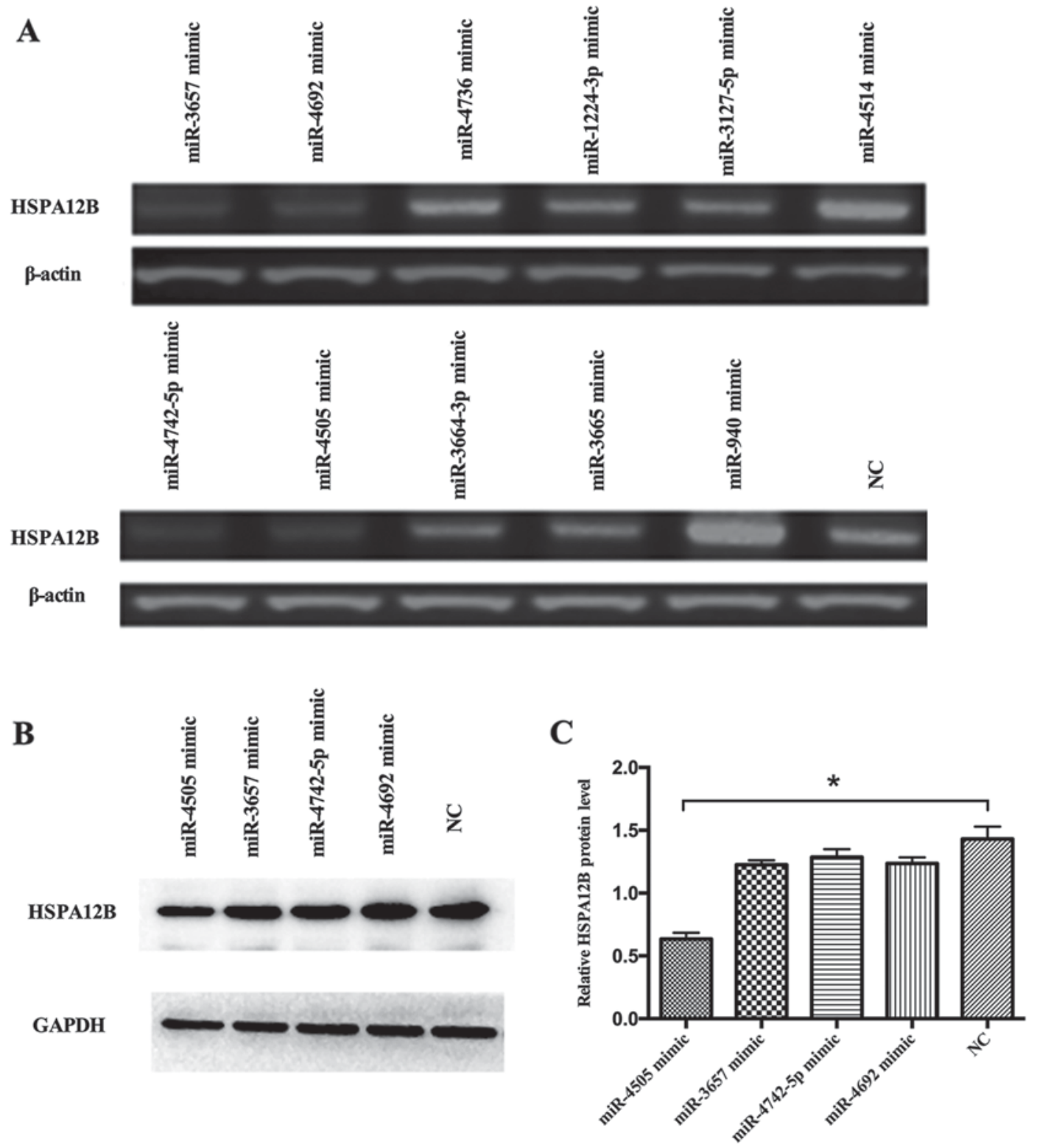

Figure 2. The expression changes of HSPA12B in HUVECs transfected with miRNA mimics. (A) HUVECs were transfected with miRNA mimics, and expression of HSPA12B mRNA was detected by RT-qPCR. (B) Expression of HSPA12B protein was measured by Western Blot. (C) Quantitative western blot analysis of the protein expression of HSPA12B with GAPDH as a loading control. The results are mean \pm standard deviation from three independent experiments. "P<0.05. HUVECs, human umbilical vein endothelial cells; HSPA12B, heat shock protein family A member 12B; miRNA, microRNA; RT-qPCR, reverse transcription quantitative polymerase chain reaction; $\mathrm{NC}$, negative control; miR, microRNA.

we designed a mutant in the HSPA12B mRNA 3'UTR that mutated eight common nucleotides in HSPA12B target site. The wild-type and mutant 3'UTR sequences of HSPA12B were shown in Fig. 3. The fluorescence ratio in HUVECs co-transfected with pGL3-HSPA12B-3'UTR plasmid and hsa-miR-4505 mimics was significant lower than in those transfected with pGL3-HSPA12B-3'UTR plasmid and negative control $(\mathrm{P}<0.05)$, but there was no significant difference between the two groups of cells co-transfected with mutant pGL3-HSPA12B-3'UTR and miR-4505 mimics or negative control (Fig. 3).

The role of $N F-\kappa B$ in miR-4505 upregulation induced by LPS. Since miR-4505 was upregulated by LPS stimulation, we studied whether NF- $\kappa \mathrm{B}$ was involved in regulating the expression of miR-4505. Cells were pretreated with NF- $\mathrm{BB}$ inhibitor and subsequently stimulated with LPS. The expression of miR-4505 was detected at 0 and $12 \mathrm{~h}$ after LPS treatment. It was shown that miR-4505 was significantly downregulated at $12 \mathrm{~h}$ after LPS stimulation in inhibitor group compared with in control group $(\mathrm{P}<0.05)$ (Fig. 4).

miR-4505 aggravates LPS-induced HUVEC injury by targeting HSPA12B. Transendothelial electrical resistance (TEER), VE-cadherin (CD144) expression and migration capacity were determined in HUVEC in order to study the effect of miR-4505 on HUVEC function. TEER assay showed that TEER was significantly lower in miR-4505 group than in the $\mathrm{NC}$ group $(\mathrm{P}<0.05)$ (Fig. 5A). Furthermore, flow cytometry suggested that VE-cadherin was significantly downregulated 
A

$\begin{array}{ll}\text { miR-4505 } & \mid \text { ' } \text { 3'..AGGCAGGGUCGGGUCGGA }^{\mid}|||||| \\ \text {Wild type HSPA12B 3' UTR } & \text { 5'...CCCAGCCA... } \\ \text { Mutant HSPA12B 3' UTR } & \text { 5'...GGAGUGAA... }\end{array}$

B

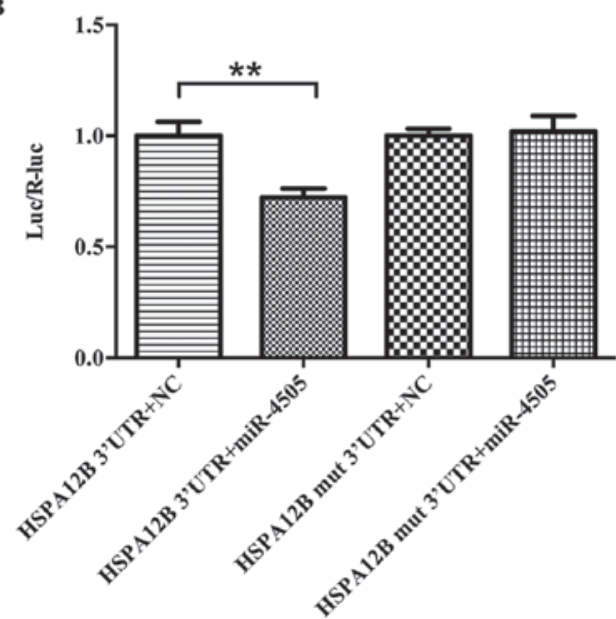

Figure 3. Dual luciferase reporter assay for the relationship between miR-4505 and the 3'UTR of HSPA12B. (A) Alignment of the predicted miR-4505 binding sites in the 3'-UTR of the HSPA12B mRNA was made. Pairing of target region (top) and miRNA (bottom) is shown. (B) Relative luciferase activity in 293 cells transfected with HSPA12B plasmids and miRNA mimics. HUVECs were co-transfected with wild-type reporter containing the HSPA12B 3'-UTR or mutant HSPA12B 3'-UTR plus miR-4505 or negative control using Invitrogen Lipofectamine 2000 reagents. Luminescence was measured after $36 \mathrm{~h}$ of transfection. The fluorescence ratio in HUVECs co-transfected with pGL3-HSPA12B-3'UTR plasmid and hsa-miR-4505 mimics was significant lower than in those transfected with pGL3-HSPA12B-3'UTR plasmid and negative control, but there was no significant difference between the two groups of cells co-transfected with mutant pGL3-HSPA12B-3'UTR and miR-4505 mimics or negative control The results are mean \pm standard deviation from three independent experiments. ${ }^{* *} \mathrm{P}<0.05$. HSPA12B, heat shock protein family A member $12 \mathrm{~B}$; UTR, untranslated region; miR, microRNA; mut, mutant; NC, negative control; luc, luciferase; HUVECs, human umbilical vein endothelial cells.

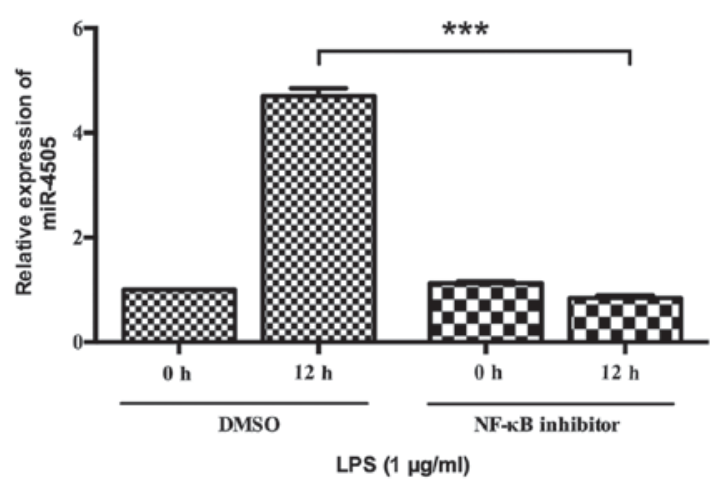

Figure 4. Effect of NF- $\kappa \mathrm{B}$ inhibitor $(1 \mu \mathrm{g} / \mathrm{ml})$ on hsa-miR-4505 expression after LPS stimulation. The expression of hsa-miR- 4505 mRNA was detected by RT-qPCR. The results are mean \pm SD from three independent experiments. ${ }^{* * *} \mathrm{P}<0.05$. DMSO, dimethyl sulfoxide; NF- $\mathrm{kB}$, nuclear factor $\kappa \mathrm{B}$; RT-qPCR, reverse transcription quantitative polymerase chain reaction; miR, microRNA; LPS, lipopolysaccharide.

after miR-4505 transfection $(\mathrm{P}<0.05)$ (Fig. 5B and $\mathrm{C})$. The scratch test showed that area of miR-4505 mimic transfection group at $0 \mathrm{~h}$ was $1.77 \pm 0.005 \mathrm{~mm}^{2}, 24 \mathrm{~h}$ was $1.38 \pm 0.1 \mathrm{~mm}^{2}$; $\mathrm{NC}$ group at $0 \mathrm{~h}$ was $1.77 \pm 0.003 \mathrm{~mm}^{2}, 24 \mathrm{~h}$ was $0.04 \pm 0.02 \mathrm{~mm}^{2}$. The migration area in the miR-4505 group $(23.4 \pm 5.7 \%)$ was significantly reduced compared with the NC group $(97.2 \pm 1.1 \%)$ $(\mathrm{P}<0.05)$ (Fig. 5D and E).

\section{Discussion}

To date, our present study demonstrated that miR-4505 negatively regulated the expression of HSPA12B by targeting the 3'UTR of HSPA12B mRNA. The expression level of hsa-miR-4505 increases after LPS stimulation in HUVECs, the mechanism may involve NF- $\mathrm{KB}$ activation. miR-4505 induces high permeability and reduces migration in LPS-induced HUVECs.

HSPA12B, as a member of the HSP70 family, is mainly expressed in endothelial cells. Our previous study showed that depletion of HSPA12B was harmful to LPS induced endothelial injury, as manifested by TEER and VE-Cadherin expression. HSPA12B protein was upregulated since $9 \mathrm{~h}$ after LPS stimulation, but returned to normal level at $24 \mathrm{~h}$. Therefore, we speculated that HSPA12B was one of the intrinsic negative-feedback mechanisms inhibiting endothelial inflammation, but some unknown reasons were inhibiting the expression of HSPA12B after the onset of inflammation. Both translational and post-translational factors might be involved in the downregulation of HSPA12B, among which miRNAs were critical for silencing protein expression. Therefore, we screened several miRNAs potentially correlated with HSPA12B by the bioinformatics analysis.

Endothelial dysfunction is a hallmark of inflammatory organ dysfunction, such as acute respiratory distress syndrome $(14,15)$. Some endothelium-derived molecules have been identified as biomarker for sepsis prognosis. For example, angiopoietin-2 was reported to be associated with respiratory failure or septic shock in several studies $(16,17)$. Our previous study also demonstrated that HSPA12B was a good predictor for death in patients with severe sepsis (18). Collapse of endothelial cells might be one of the causes of the secretion of these proteins. Thus, protecting endothelial cells might be a promising direction for improving sepsis or acute respiratory distress syndrome.

Endothelial function was partially manifested by the permeability and migration. During inflammation response, increased endothelial permeability triggers the body fluids accumulating, leads to pulmonary edema and organ dysfunction (19). Vascular endothelial cells and extracellular components (such as glycocalyx) are important regulators of pulmonary vascular barrier (20). VE-cadherin is a component of endothelial junctions, and plays a key role in the maintenance of vascular integrity (21). Several studies showed that monolayer endothelial cell could increase the endothelium permeability via VE-Cadherin antibody (22). VE-cadherin antibody administration can cause pulmonary and cardiac edema in mice $(23,24)$. In sepsis, high expression of cytokines (TNF- $\alpha$, vascular endothelial growth factor) could redistribute VE-Cadherin focally and increase the endothelium permeability. Therefore, our study demonstrated that miR-4505 aggravated endothelial injury by targeting at HSPA12B. 
A

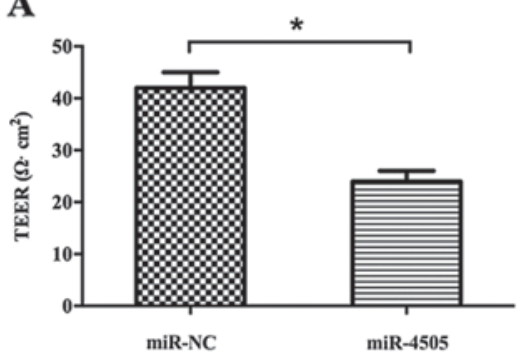

D
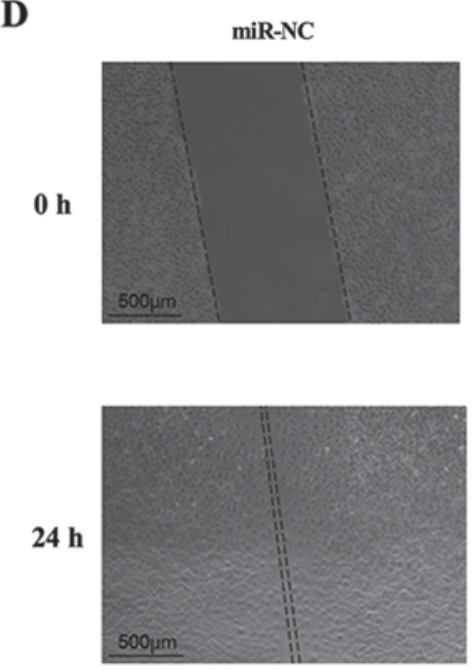

B

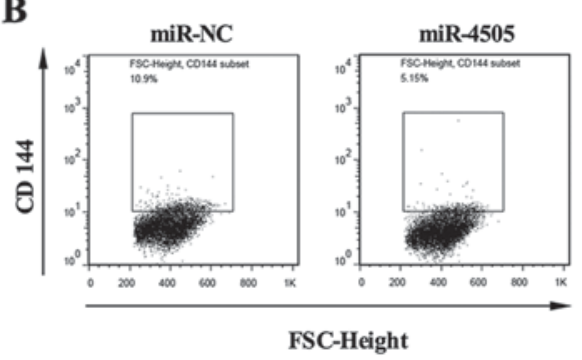

C

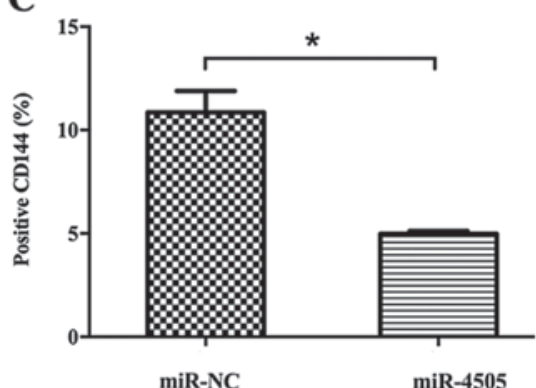

$\mathbf{E}$

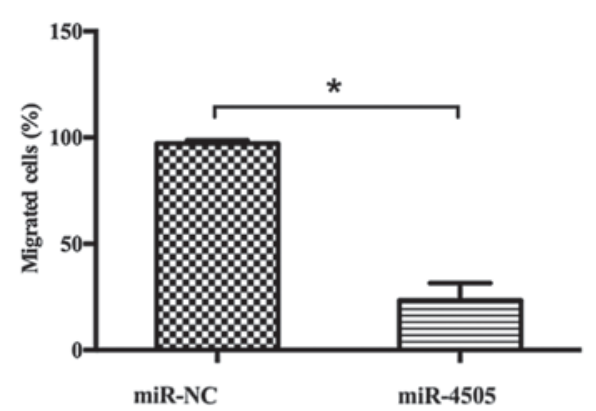

Figure 5. Hsa-miR-4505 aggravated LPS-induced HUVEC injury by targeting HSPA12B. (A) Permeability of HUVEC transfected with miR-4505 mimics or NC after LPS challenge, as shown by TEER values. (B and C) Flow cytometry assay of VE-Cadherin in HUVECs. (D and E) Scratch test following transfection with miR-4505 mimics in HUVECs. The results are mean \pm SD from three independent experiments. *P<0.05. LPS, lipopolysaccharide; HUVECs, human umbilical vein endothelial cells; NC, negative control; miR, microRNA; CD, cluster of differentiation; VE-cadherin, vascular endothelial cadherin; miR, microRNA; TEER, transepithelial electrical resistance.

In summary, miR-4505 negatively regulates HSPA12B expression via direct interaction with the 3'UTR of HSPA12B mRNA. Upregulation of miR-4505 by LPS challenge was dependent on NF- $\mathrm{kB}$. Overexpression of miR-4505 aggravated endothelial injury as shown by the increased permeability and reduce migration capacity. However, there are several limitations in our present study. First, the study was carried out in HUVECs, we have no data in vivo about miRNA-4505. To evaluate the efficacy of miR-4505 in HSPA12B-mediated protective effect on endothelial cells following LPS challenge, a more comprehensive study in vivo may be necessary to validate our findings. Second, the expression of miR-4505 in plasma of sepsis patients remains to be measured. Further experiments need to be performed to determine whether the miR-4505 negatively regulates HSPA12B expression in sepsis patients.

\section{Acknowledgements}

This work was supported by the grants from the National Natural Science Foundation of China (No. 81270128). The manuscript partly presented in the 10th National Assembly severe medical National Medical Conference 2016 as the form of poster presentation (http://www.medmeeting.org/Upload/user/186151/file/2 0160520/20160520095931_8385.pdf).

\section{References}

1. Singer M, Deutschman CS, Seymour CW, Shankar-Hari M, Annane D, Bauer M, Bellomo R, Bernard GR, Chiche JD, Coopersmith CM, et al: The third international consensus definitions for sepsis and septic shock (Sepsis-3). JAMA 315: 801-810, 2016.

2. Hernu R, Wallet F, Thiollière F, Martin O, Richard JC, Schmitt Z, Wallon G, Delannoy B, Rimmelé T, Démaret C, et al: An attempt to validate the modification of the American-European consensus definition of acute lung injury/acute respiratory distress syndrome by the Berlin definition in a university hospital. Intensive Care Med 39: 2161-2170, 2013.

3. Parikh SM, Mammoto T, Schultz A, Yuan HT, Christiani D, Karumanchi SA and Sukhatme VP: Excess circulating angiopoietin-2 may contribute to pulmonary vascular leak in sepsis in humans. PLoS Med 3: e46, 2006.

4. Stevens T: Functional and molecular heterogeneity of pulmonary endothelial cells. Proc Am Thorac Soc 8: 453-457, 2011.

5. Steagall RJ, Rusiñol AE, Truong QA and Han Z: HSPA12B is predominantly expressed in endothelial cells and required for angiogenesis. Arterioscler Thromb Vasc Biol 26: 2012-2018, 2006.

6. Zhou H, Qian J, Li C, Li J, Zhang X, Ding Z, Gao X, Han Z, Cheng Y and Liu L: Attenuation of cardiac dysfunction by HSPA12B in endotoxin-induced sepsis in mice through a PI3K-dependent mechanism. Cardiovasc Res 89: 109-118, 2011.

7. Li J, Zhang Y, Li C, Xie J, Liu Y, Zhu W, Zhang X, Jiang S, Liu L and Ding Z: HSPA12B attenuates cardiac dysfunction and remodelling after myocardial infarction through an eNOS-dependent mechanism. Cardiovasc Res 99: 674-684, 2013. 
8. Wu J, Li X, Huang L, Jiang S, Tu F, Zhang X, Ma H, Li R, Li C, Li Y, et al: HSPA12B inhibits lipopolysaccharide-induced inflammatory response in human umbilical vein endothelial cells. J Cell Mol Med 19: 544-554, 2015.

9. Kang Q, Chen Y, Zhang X, Yu G, Wan X, Wang J, Bo L and Zhu K: Heat shock protein A12B protects against sepsis-induced impairment in vascular endothelial permeability. J Surg Res 202: 87-94, 2016.

10. Meltzer PS: Cancer genomics: Small RNAs with big impacts. Nature 435: 745-746, 2005

11. Lee Y, Jeon K, Lee JT, Kim S and Kim VN: MicroRNA maturation: Stepwise processing and subcellular localization. EMBO J 21: 4663-4670, 2002.

12. Babiarz JE, Ruby JG, Wang Y, Bartel DP and Blelloch R: Mouse ES cells express endogenous shRNAs, siRNAs, and other microproces-sor-independent, Dicer-dependent small RNAs. Genes Dev 22: 2773-2785, 2008.

13. O'Connell RM, Rao DS, Chaudhuri AA and Baltimore D: Physiological and pathological roles for microRNAs in the immune system. Nat Rev Immunol 10: 111-122, 2010.

14. Coletta C, Módis K, Oláh G, Brunyánszki A, Herzig DS, Sherwood ER, Ungvári Z and Szabo C: Endothelial dysfunction is a potential contributor to multiple organ failure and mortality in aged mice subjected to septic shock: Preclinical studies in a murine model of cecal ligation and puncture. Crit Care 18: 511,2014

15. Lee WL and Liles WC: Endothelial activation, dysfunction and permeability during severe infections. Curr Opin Hematol 18: 191-196, 2011.

16. Palud A, Parmentier-Decrucq E, Pastre J, De Freitas Caires N, Lassalle $\mathrm{P}$ and Mathieu D: Evaluation of endothelial biomarkers as predictors of organ failures in septic shock patients. Cytokine 73: 213-218, 2015.
17. Fang Y, Li C, Shao R, Yu H, Zhang Q and Zhao L: Prognostic significance of the angiopoietin-2/angiopoietin-1 and angiopoietin-1/Tie-2 ratios for early sepsis in an emergency department. Crit Care 19: 367, 2015

18. Zhang R, Wan XJ, Zhang X, Kang QX, Bian JJ, Yu GF, Wang JF and Zhu KM: Plasma HSPA12B is a potential predictor for poor outcome in severe sepsis. PLoS One 9: e101215, 2014.

19. Birukov KG, Zebda N and Birukova AA: Barrier enhancing signals in pulmonary edema. Compr Physiol 3: 429-484, 2013.

20. Yang Y and Schmidt EP: The endothelial glycocalyx: An important regulator of the pulmonary vascular barrier. Tissue Barriers 1: pii: 23494, 2013.

21. Giannotta M, Trani M and Dejana E: VE-cadherin and endothelial adherens junctions: Active guardians of vascular integrity. Dev Cell 26: 441-454, 2013.

22. Breviario F, Caveda L, Corada M, Martin-Padura I, Navarro P, Golay J, Introna M, Gulino D, Lampugnani MG and Dejana E: Functional properties of human vascular endothelial cadherin (7B4/cadherin-5), an endothelium-specific cadherin. Arterioscler Thromb Vasc Biol 15: 1229-1239, 1995.

23. Gotsch U, Borges E, Bosse R, Böggemeyer E, Simon M, Mossmann $\mathrm{H}$ and Vestweber D: VE-cadherin antibody accelerates neutrophil recruiment in vivo. J Cell Sci 110: 583-588, 1997.

24. Corada M, Mariotti M, Thurston G, Smith K, Kunkel R and Brockhaus M: Vascular endothelial-cadherin is an important determinant of microvascular integrity in vivo. Proc Natl Acad Sci USA 96: 9815-9820, 1999. 